\title{
Supermodel Analysis of the Hard X-Ray Excess in the Coma Cluster
}

\author{
R. Fusco-Femiano ${ }^{1}$, M. Orlandini ${ }^{2}$, M. Bonamente ${ }^{3,4}$ and A. Lapi ${ }^{5,6}$
}

\begin{abstract}
The Supermodel provides an accurate description of the thermal contribution by the hot intracluster plasma which is crucial for the analysis of the hard excess. In this paper the thermal emissivity in the Coma cluster is derived starting from the intracluster gas temperature and density profiles obtained by the Supermodel analysis of X-ray observables: the XMM-Newton temperature profile and the ROSAT brightness distribution. The Supermodel analysis of the BeppoSAX /PDS hard X-ray spectrum confirms our previous results, namely an excess at the c.l. of $\sim 4.8 \sigma$ and a nonthermal flux of $1.30 \pm 0.40 \times 10^{-11} \mathrm{erg} \mathrm{cm}^{-2} \mathrm{~s}^{-1}$ in the energy range $20-80 \mathrm{keV}$. A recent joint XMM-Newton / Suzaku analysis reports an upper limit of $\sim 6 \times 10^{-12} \mathrm{erg} \mathrm{cm}^{-2} \mathrm{~s}^{-1}$ in the energy range $20-80 \mathrm{keV}$ for the nonthermal flux with an average gas temperature of $8.45 \pm 0.06 \mathrm{keV}$, and an excess of nonthermal radiation at a confidence level above $4 \sigma$, without including systematic effects, for an average XMM-Newton temperature of $8.2 \mathrm{keV}$ in the Suzaku /HXD-PIN FOV, in agreement with our earlier PDS analysis. Here we present a further evidence of the compatibility between the Suzaku and BeppoSAX spectra, obtained by our Supermodel analysis of the PDS data, when the smaller size of the HXD-PIN FOV and the two different average temperatures derived by XMMNewton and by the joint $X M M-N e w t o n / S u z a k u$ analysis are taken into account. The consistency of the PDS and HXD-PIN spectra reaffirms the presence of a nonthermal component in the hard X-ray spectrum of the Coma cluster. The Supermodel analysis of the PDS data reports an excess at c.l. above $4 \sigma$ also for the higher average temperature of $8.45 \mathrm{keV}$ thanks to the PDS FOV considerably greater than the HXD-PIN FOV.
\end{abstract}

Subject headings: galaxies: clusters: general — galaxies: clusters: individual (Coma) - intergalactic medium — radiation mechanisms: non-thermal - X-rays: galaxies: clusters.

\footnotetext{
${ }^{1}$ INAF/IASF-Roma, via del Fosso del Cavaliere, I-00133 Roma, Italy

${ }^{2}$ INAF/IASF-BO, via Gobetti 101, I-40129 Bologna, Italy

${ }^{3}$ Department of Physics, University of Alabama, Huntsville, AL35899

${ }^{4}$ National Space Science and Technology Center, NASA Marshall Space Flight Center, Huntsville, AL35812

${ }^{5}$ Dip. Fisica, Univ. 'Tor Vergata', Via Ricerca Scientifica 1, I-00133 Roma, Italy

${ }^{6}$ SISSA/ISAS, Via Bonomea 265, I-34136 Trieste, Italy
} 


\section{Introduction}

The Supermodel (SM) describes the density and temperature profiles when we consider the entropy-modulated equilibrium of the intracluster plasma (ICP) within the potential wells provided by the dominant Dark Matter (DM). These two components are related not only by their common potential well but also by parallel accretion of surrounding DM and baryons into the cluster volume (Cavaliere, Lapi \& Fusco-Femiano 2009)1.

An analysis of the X-ray brightness and temperature profiles for both cool core (CC) and non cool core (NCC) clusters has been performed in terms of the SM (Cavaliere, Lapi \& FuscoFemiano 2009; Fusco-Femiano, Cavaliere \& Lapi 2009; thereafter FFCL09; Lapi, Fusco-Femiano \& Cavaliere 2010). This analysis highlights how simply the SM represents the main dichotomy "CC versus NCC" clusters in terms of a few ICP parameters governing the radial entropy run $(k(r)=$ $T(r) / n(r)^{2 / 3}$, where $T(r)$ and $n(r)$ are the ICP temperature and density profiles, respectively) and shows how accurately it fits even complex brightness and temperature profiles. This dichotomy can be represented and understood in terms of two physical parameters marking the ICP entropy profile: the central value $k_{c}$ and the outer slope $a$. More structured temperature and brightness profiles need an additional, physical parameter $r_{f}$ marking the extension of the entropy floor.

The SM has shown that the inward decline of the temperature profile $T(r)$ characteristic of CC clusters is a feature of the non-radiative SM equilibrium focusing also the conditions for a cooling catastrophe that may be stabilized by ICP condensing around and into a central massive galaxy to trigger accretion on the nuclear black hole. The feature common to $\mathrm{CC}$ clusters is their low values of $a \leq 1$ and their high values of the concentration $c>4$ that follow from their being old structures. At the other extreme, the NCC clusters appear to be dynamically young structures characterized by high values of $a$ and low concentrations. The central flat brightness profile present in NCC clusters like Coma and A2256 reveals large central injections of energy and entropy deposited in the form of a floor extended out to $r_{f}$. The SM challenges the complexity posed by substructures observed in the temperature profiles of A2256 and A644. It evidences the existence of cold regions that are remnants of a previous cool phase partially erased by a merger event. Such cases may be termed as RCCs for remnant of CCs. Recently, the SM analysis (Lapi, Fusco-Femiano \& Cavaliere 2010) of the steep temperature declines in CC clusters at low redshift (A1795 and PKS 0745-191) observed by Suzaku requires a progressive flattening of the entropy run starting at $r \gtrsim 0.2$ of the virial radius $R$ in agreement with the reported entropy profiles (Bautz et al. 2009; George et al. 2009). Lapi, Fusco-Femiano \& Cavaliere (2010) argue that the entropy production at the cluster boundary is reduced or terminated as the accretion rates of DM and intergalactic gas peter out. This weakening of the accretion shocks demands turbulence to develop also in the outskirts of relaxed clusters (Cavaliere, Lapi \& Fusco-Femiano 2011).

\footnotetext{
${ }^{1}$ The interested reader may try for her/himself to use the fast SM algorithm made available at the website http://people.sissa.it/ lapi/Supermodel/
} 
BeppoSAX detected the presence of non thermal (NT) radiation in excess of the thermal ICP emission in the Coma cluster (Fusco-Femiano et al. 1999; 2004) and Abell 2256 (Fusco-Femiano et al. 2000; Fusco-Femiano, Landi \& Orlandini 2005). This evidence has been claimed also by RXTE observations (Rephaeli, Gruber \& Blanco 1999; Rephaeli \& Gruber 2002; 2003) reporting NT fluxes in the 20-80 keV energy band in agreement with the BeppoSAX values. The PDS onboard BeppoSAX was a suitable instrument to detect NT radiation in galaxy clusters. Since clusters are very weak sources at $\mathrm{HXR}$ energies above $15 \mathrm{keV}$ a correct determination of the background is crucial. Thanks to the rocking technique, the PDS was able to perform a background measurement simultaneously with the observations and therefore no modelling of the background was necessary, as is required for other detectors. Moreover, the background was very stable and low for the equatorial orbit of BeppoSAX .

For the Coma cluster the PDS analysis has been challenged by the analysis of Rossetti \& Molendi (2004, thereafter RM04) with a different software package (SAXDAS) instead of XAS. In 2007 Fusco-Femiano, Landi \& Orlandini (thereafter FFLO07) have demonstrated that the use of the SAXDAS package allows to obtain the same results of the previous analysis with XAS (Fusco-Femiano et al. 2004, thereafter FF04). The main reason of the discrepancy between the two analyses is in the non accurate selection of the events by RM04 and not in the treatment of the total background as reported by Wik et al. (2011). In particular, an incorrect handling of spurious spikes due to environmental background, when present, introduces noise that enlarges the error bars hiding the presence of a NT excess over the thermal radiation. In fact, FFLO07 show that the c.l. of the excess increases from $2.9 \sigma$ to $4.2 \sigma$ when the same time windows of XAS analysis are considered in SAXDAS. Unfortunately, this important point is not reported in the review of Rephaeli et al. (2008). Additional differences between the two analyses that lead to a c.l. of $4.8 \sigma$ for the excess and the remarks, including the systematic effects, reported in RM04 are amply discussed in FFLO07. Moreover, we underline that the consistency of the cosmic hard X-ray background measured by BeppoSAX/PDS (Frontera et al. 2007) with the spectrum observed by Integral (Churazov et al. 2007; Bisnovatyi-Kogan \& Pozanenko 2010) and Swift (Ajello et al. 2008), all comparable with the historic HEAO-1 measurements (Gruber et al. 1999), implies negligible PDS systematic effects as reported in FFLO07 and Frontera et al. (2007). The correctness of the PDS analyses is also evidenced by the agreement between the BeppoSAX /PDS and Suzaku /HXD-PIN spectra for the cluster Abell 3667 (Fusco-Femiano et al. 2001; Nakazawa et al. 2009).

Suzaku observations (Wik et al. 2009, thereafter W09) constrain the thermal component by the hot ICP using a joint XMM-Newton \& Suzaku /HXD-PIN analysis reporting an upper limit of $\sim 6 \times 10^{-12} \mathrm{erg} \mathrm{cm}^{-2} \mathrm{~s}^{-1}$ in the energy range $20-80 \mathrm{keV}$ for the NT emission with an average temperature of $8.45 \pm 0.06 \mathrm{keV}$. Also, they found an excess at c.l. above $4 \sigma$ with an annular $X M M$ Newton best-fit value of $8.2 \mathrm{keV}$ in the Suzaku /HXD-PIN FOV, in agreement with the results of FF04. For the lower temperature W09 do not report the NT flux value that this SM analysis indicates to be $\sim 20 \%$ lower than the PDS NT flux here reported for the smaller HXD-PIN FOV than the PDS FOV (see Sect.s 2 and 3). 
With our SM analysis we will show that the marginal evidence of a NT component in the Suzaku observations is due to two combined causes: loss of NT flux for the smaller FOV of the HXD-PIN with respect to the BeppoSAX /PDS and RXTE FOVs, and higher average temperature derived by the joint analysis.

The Coma cluster has been observed also by Integral (Eckert et al. 2007; Lutovinov et al. 2008) and Swift /BAT (Ajello et al. 2009). Eckert et al. (2007) explore the morphology of the cluster in the HXR energy range 18-30 keV with a deep observation. The Integral image is displaced in direction of the NGC 4839 group which is merging with the main cluster. They associate the HXR excess in this region with emission from a very hot region of the cluster $(T \geqslant 10 \mathrm{keV})$ showed by Neumann et al. (2003) in their XMM-Newton temperature map.

Combining data from Integral, RXTE and ROSAT observatories, Lutovinov et al. (2008) find that the thermal spectrum can be modelled with a temperature of $8.2 \mathrm{keV}$ and that the cluster is only marginally detectable $(\sim 1.6 \sigma)$ in the $44-107 \mathrm{keV}$ energy band by Integral . The $20-80$ $\mathrm{keV}$ flux of a possible NT component $\left(6.0 \pm 8.8 \times 10^{-12} \mathrm{erg} \mathrm{cm}^{-2} \mathrm{~s}^{-1}\right)$ is consistent with the $B e p p o S A X$ and RXTE fluxes. They also exclude with high significance that the NT emission reported by BeppoSAX and $R X T E$ could be due to a single point source.

The Swift mission is mainly devoted to detect and localize gamma-ray bursts. Swift BAT is a coded-mask telescope optimized for the study of point-like sources and can be used to investigate extended objects only if these are detected as point-like. Coma instead is extended in BAT and part of the source flux is lost in the BAT background. Ajello et al. (2009) treated the Coma cluster as a point-like source considering source emission within a radius of $\sim 10^{\prime}$ from the BAT centroid. They conclude that the presence of a NT component arising from the cluster outskirts cannot be excluded. More recently, Wik et al. (2011) have tested the possibility that the difference between the Suzaku /HXD-PIN upper limit (for $T=8.45 \mathrm{keV}$ ) and the BeppoSAX and RXTE NT fluxes may be due to the extent of the inverse Compton (IC) emission. Their joint XMM-Newton / Swift BAT analysis requires an accurate cross-calibration between the two instruments and to model both the thermal and NT spatial distributions. Moreover, the analysis is characterized by a higher uncertainty than for a point source (Ajello et al. 2009; Wik et al. 2011). The derived upper limits to the NT radiation are inconsistent with the BeppoSAX and RXTE observations.

In this paper, Sect. 2 reports the procedure followed for the SM analysis of the HXR PDS spectrum in the Coma cluster. This analysis relies on the ICP density and temperature profiles fixed by the SM analysis (FFCL09) of the ROSAT X-ray brightness and XMM-Newton temperature distributions. The presence of a NT spectral component in the HXR PDS spectrum is identified by determining in any point of the cluster the thermal ICP emissivity at a given energy. In the previous BeppoSAX and RXTE analyses the ICP thermal contribution was estimated considering bremsstrahlung emission for an average temperature in the FOV of the instruments. Sect.s 3 and 4 are devoted to the discussion and conclusions, respectively.

In our treatment, we adopt a Coma cluster redshift of 0.0232 , Hubble constant $H_{0}=70 \mathrm{~km} \mathrm{~s}^{-1} \mathrm{Mpc}^{-1}$, 
and quote errorbars at the $68 \%$ confidence level. One arcmin corresponds to $28.12 \mathrm{kpc}$.

\section{SM Analysis of the Hard Excess}

The SM analysis of the Coma cluster (FFCL09) involves the fit to the XMM-Newton projected emission-weighted temperature profile (Snowden et al. 2008) and to the ROSAT surface brightness distribution (Mohr et al. 1999) (see Fig. 1). For this paper we have performed a slight different SM analysis of the X-ray brightness profile with respect to that in FFCL09. We imposed the same value of $r_{f}=96 \pm 5 \mathrm{kpc}$ derived by the temperature profile obtaining a very good fit to the brightness profile (see Fig. 1). Instead, a not acceptable $\chi^{2}$ value is obtained imposing $r_{f}=250_{-74}^{+44}$ $\mathrm{kpc}$, derived by the previous analysis of the brightness profile (see FFCL09), in the fit to the temperature distribution. This implies that the temperature profile is more accurate than the brightness profile in the determination of the entropy floor extension $r_{f}$. From this new analysis we obtain a value of $4.3 \pm 0.4 \times 10^{-5} \mathrm{~cm}^{-3}$ for the density at the virial radius $R$. Thus, the values of $r_{f}$ and $n_{R}$ are slight different from those reported in FFCL09, while the ICP temperature at the virial radius is $T_{R}=5.7 \pm 1.0 \mathrm{keV}$ as reported in FFCL09.

Summarizing, the free parameter values that fit the emission-weighted temperature profile of Fig. 1 and that determine the temperature and density profiles of Fig. 2 are: $c=1.67^{+4.30}$ for the $\mathrm{DM}$ and: $k_{c} / k_{R}=(1.14 \pm 0.83) \times 10^{-1}, a=1.03^{+0.77}$ and $r_{f} / R=(4.37 \pm 0.23) \times 10^{-2}$ for the ICP. These values fit the ROSAT brightness profile (see Fig. 1) when they vary within their associated errors. The inverse process that implies to derive the parameter values from the brightness profile to fit the temperature distribution is not adequate for the weak dependence of the entropy on the brightness $B\left(k=T / n^{2 / 3} \sim T^{7 / 6} / B^{1 / 3}\right.$ where $\left.B \sim n^{2} T^{1 / 2}\right)$.

While the use of ROSAT data is not justified in the central regions by the PSPC angular resolution $\left(\sim 25^{\prime \prime}\right)$ with respect to a XMM-Newton profile, at larger radii the latter suffers of a greater total background. Vikhlinin et al. (2006) find an excellent agreement between Chandra and ROSAT PSPC surface brightness data at large distances where the ROSAT data allow to have a better statistical accuracy.

Several determinations of the virial radius are given in the literature ranging between 2 and 3 Mpc (Castander et al. 2001; Lokas \& Mamon 2003; Kubo et al. 2007; Gavazzi et al. 2009). A value of 2.2 Mpc (Gavazzi et al. 2009) has been adopted that corresponds to 78.24'. The results of the SM analysis (FFCL09) depend only weakly on this choice within one standard deviation.

The profiles of Fig. 1 correspond to the temperature and density profiles of Fig. 2, with a central temperature of $9.65 \mathrm{keV}$ and central density of $2.5 \times 10^{-3} \mathrm{~cm}^{-3}$. We highlight that the projected emission-weighted temperature SM profile of Fig. 1 gives a value of $8.21 \pm 0.08 \mathrm{keV}$ (90\% c.l.) in the Suzaku FOV $\left(34^{\prime} \times 34^{\prime}\right)$, the same value found by W09 in their spectral fits to the $X M M$-Newton regions of the Coma cluster and in agreement with previous measurements. Hughes et al. (1993) derive 8.21 \pm 0.16 (90\% c.l. and including systematic effects) from Ginga over the energy 
range from 1.5 to $20 \mathrm{keV}$ (collimator $1^{\circ}-2^{\circ} \mathrm{FWHM}$ ) and Arnaud et al. (2001) report 8.25 \pm 0.10 $\mathrm{keV}\left(90 \%\right.$ c.l.) in the central $r<10^{\prime}$ region with the XMM-Newton-EPIC-MOS detectors (energy range $0.3-10 \mathrm{keV})$.

To check the SM extrapolation to the virial radius of the temperature profile represented by the dashed curve of Fig. 1 we derive the average temperature within the single collimator with a total square FOV of $65.5^{\prime}$ on a side considered by W09 to approximate the HXD-PIN spatial response. Our SM value of $\sim 7.85 \mathrm{keV}$ is consistent with the temperature values, reported in Table 1 of W09 in their XMM-Newton analysis of the Coma cluster regions, that give an average temperature of $7.79 \pm 0.12 \mathrm{keV}$. This agreement is also visible in Fig. 3 that reports the temperature values in the $X M M$-Newton regions R1-R6 investigated by W09 showing a temperature decline consistent with our SM extrapolation up to a distance of more than $30^{\prime}(\sim 0.4 R)$ from the cluster center.

To compute the X-ray emission spectrum of the Coma cluster we consider the MEKAL plasma model (Mewe, Gronenschild, \& van den Oord 1985; Mewe, Lemen, \& van den Oord 1986; Kaastra 1993), the Galactic absorption model (Morrison \& McCammon 1981), and the abundance profile $Z(r)$ of Leccardi et al. (2010) for NCC clusters. To take into account the temperature $T(r)$ and density $n(r)$ profiles of Fig. 2 in SM analysis of the PDS spectrum we utilize the routine xsmekl outside of the $X S P E C$ package. At energies above $50 \mathrm{keV}$ where the MEKAL model is undefined we use Eq. 1b of Mewe et al. (1986) to derive the photon number emissivity at energy $E$ per unit energy interval. The emissivity computed in any point of the cluster $F(E)=n^{2}(r) \Lambda[T(r), Z(r)]$ in photons $\mathrm{cm}^{-3} \mathrm{~s}^{-1}$ is projected along the line-of-sight for each energy and then integrated between a spatial interval $\left(r_{1}-r_{2}\right)$ always for each energy. $\Lambda$ is the emissivity derived by xsmekl. The Coma cluster flux in photons $\mathrm{cm}^{-2} \mathrm{~s}^{-1}$ represents an additive model that through the command model atable in XSPEC fits the data.

The ICP temperature and density profiles of Fig. 2 determine the cluster thermal emissivity in the energy range 15-80 keV. To compare the SM spectrum with the PDS spectrum (FF04) we have integrated the projected emissivity at a given energy in the full BeppoSAX FOV,$r_{2}=78^{\prime}\left(r_{1}=0^{\prime}\right)$. The SM thermal flux at $15 \mathrm{keV}$ results to be lower by a factor 1.11 than the PDS flux at the same energy implying a NT excess even at $15 \mathrm{keV}$. Considering the calibration offset between the ROSAT / XMM-Newton fit and the BeppoSAX data (see Kirsch et al. 2005) we have conservatively normalized the SM thermal spectrum to the PDS flux observed at $15 \mathrm{keV}$. This requires a slight increase of $n_{R}$, at $\sim 4.5 \times 10^{-5} \mathrm{~cm}^{-3}$ that is within the $1 \sigma$ uncertainty of the SM determination. After the normalization, we still detect a NT component at $E \geq 20 \mathrm{keV}$ with significance $\sim 4.8 \sigma$ with a flux in the energy range $20-80 \mathrm{keV}$ of $1.30 \pm 0.40 \times 10^{-11} \mathrm{erg} \mathrm{cm}^{-2} \mathrm{~s}^{-1}$ for an assumed photon index $\Gamma=2$ (see Fig. 4). The significance and the flux of the NT component are consistent with the previous analysis of FF04 ( $\sigma=4.8$ and flux of $\left.1.5 \pm 0.5 \times 10^{-11} \mathrm{erg} \mathrm{cm}^{-2} \mathrm{~s}^{-1}\right)$ using an average temperature of $8.11 \mathrm{keV}$ derived by Ginga (David et al. 1993). This result is also in agreement with the NT component significance greater than $4 \sigma$, without including systematic effects, obtained by Suzaku (W09) for $T=8.2 \mathrm{keV}$, the same temperature obtained by the SM for the Suzaku FOV (see Fig. 1). The NT origin of the hard excess has been verified fitting the PDS data with a 
thermal component, instead of a power law, in addition to the SM thermal contribution. The best-fit value for the temperature is $\sim 28.5 \mathrm{keV}$ with a lower limit of $\sim 11.5 \mathrm{keV}$ (90\% c.l.) making unlikely that the hot regions reported by XMM-Newton (Neumann et al. 2003) and Integral (Eckert et al. 2007) observations can be responsible for the hard tail detected by BeppoSAX/PDS. A recent joint analysis XMM-Newton / Swift BAT (Wik et al. 2011) has shown inconsistency between the NT upper limits derived for different spatial models with the BeppoSAX and RXTE detections. We believe that a coded-mask telescope devoted mainly to the study of point sources finds several difficulties to disentangle a NT component from the ICP thermal radiation in an extended and weak source at HXR energies like the Coma cluster (see Ajello et al. 2009). Moreover, this analysis requires an accurate cross-calibration between the two detectors.

\section{Discussion}

Extended radio regions observed in several galaxy clusters, radio halos and relics, provides evidence for the presence of a population of relativistic electrons and magnetic fields in the ICP (see Ferrari et al. 2008). The detection of NT radiation in HXR spectra imposes further constraints to the possible acceleration mechanisms and origin of the relativistic electrons responsible for NT phenomena in galaxy clusters (e.g., Brunetti et al. 2001). The likely origin of the hard excess is IC scattering of relativistic electrons by the cosmic microwave background (CMB) photons. In this scenario the volume-averaged magnetic field strength and the density of the relativistic electrons can be determined combining radio and NT X-ray fluxes (Rephaeli 1979). The Coma cluster exhibits a central giant radio halo and a peripheral radio relic with total extent of about $\sim 67^{\prime}$ at 1.41 $\mathrm{GHz}$ with a centre $75^{\prime}$ offset with respect to the X-ray cluster center. Besides, the very extended $\left(\sim 135^{\prime}\right)$ low surface radio brightness envelope first seen by Kronberg et al. $(2007)$ and confirmed by Brown \& Rudnick (2010) could be an additional source for relativistic electrons responsible for the hard IC emission observed by BeppoSAX and RXTE .

One of the most sensitive points in the search for NT components is the lack of information on the thermal structure of the hot ICP. In the analysis of the non-imaging BeppoSAX and RXTE observations it was only possible to consider an average temperature in the FOV of the instruments to determine the bremsstrahlung emissivity of the hot gas. Waiting for telescopes able to map the HXR emission and disentangle the thermal and the NT components (like NuStar 2 and Astro- $\mathrm{H}^{3}$ ), we believe that the SM is a powerful tool to constrain the ICP thermal radiation for a confident assessment of the presence of NT spectral components also in clusters like Coma that evidences ongoing mergers, hallmark of a recent cluster formation. The extension of the entropy floor, $r_{f}$, is interpreted in terms of the stallation radius attained by a outbound blast triggered by a major head-on merger or driven by a violent AGN outburst before being degraded into adiabatic sound

\footnotetext{
${ }^{2}$ http://www.nustar.caltech.edu/

${ }^{3}$ http://astro-h.isas.jaxa.jp/
} 
waves. This interpretation relates $r_{f}$ to the dating of the merger responsible for the energy/entropy input; the good performance of the SM implies such a time to be intermediate between the blast transit time $<0.1$ Gyr to reach $r_{f} \sim 100 \mathrm{kpc}$ (see Cavaliere \& Lapi 2006) and the time of several Gyrs needed by radiative cooling to erode the entropy floor. Such a timing guarantees an accurate description of the ICP thermodynamic state by the SM based on hydrostatic equilibrium (for a more detailed discussion see Sect. 5 of FFCL09). Moreover, the equilibrium of the ICP is somewhat faster to attain the DM's (see Ricker \& Sarazin 2001; Lapi et al. 2005) and the circularized data (integrated over annuli; see Snowden et al. 2008) tend to smooth out local, limited deviations from spherical hydrostatics and to better agree with equilibrium. Conditions of disequilibrium are present in clusters like the Bullet cluster (see Clowe et al. 2006) or MACS J0025.4-1222 (see Bradač et al. 2008). These conditions, due to stronger if rarer energy injections by deep major mergers, prevent a SM description of the X-ray observables.

The SM allows to determine more correctly the thermal ICP contribution than the temperature maps that are limited in extension $(\lesssim R / 2)$, except for a handful of clusters observed by Suzaku (Bautz et al. 2009; George et al. 2009; Reiprich et al. 2009; Kawaharada et al. 2010; Hoshino et al. 2010) that does not include Coma, while the BeppoSAX FOV extends to $\sim R$. Finally, the accurate SM fit to the brightness profile (see Fig. 1) implies that the relevance of the cluster ellipticity is mild.

To evidence the presence of a NT feature, we considered the SM ICP temperature and density profiles to derive the underlying contribution of the hot ICP to the HXR Coma spectrum observed by BeppoSAX /PDS in the energy range 15-80 keV. The profiles of Fig. 2 have been obtained by the SM analysis of the X-ray observables of the Coma cluster: the XMM-Newton projected emission-weighted temperature (Snowden et al. 2008) and the ROSAT brightness distribution in the energy range 0.5-2 keV (Mohr et al. 1999). Notice that the SM extrapolation of the temperature profile is in agreement with the more recent analysis of the XMM-Newton data by W09 up to a distance of $\sim 30^{\prime}$ from the cluster center (see Fig. 3), lending additional support to the use of the SM profiles. A further check of the validity of the SM profiles of Fig. 2 is given by the fit to the ROSAT PSPC spectrum (energy range 1-2 keV) in the region $20^{\prime}-40^{\prime}$ around the center of the Coma cluster (Bonamente et al. 2003). The SM spectrum is lower than the ROSAT data by only a factor $\sim 1.06$ (see Fig. 5).

The joint XMM-Newton / Suzaku HXD-PIN analysis (W09) derives a mean temperature of 8.45 $\mathrm{keV}$ in the HXD-PIN FOV greater than the temperature used in the BeppoSAX and RXTE analyses. The authors suggest that the lower temperature may be the effect of the larger FOVs of the two X-ray detectors that include emission from more cool gas in the cluster outskirts. This emission that lowers the average temperature is determined mainly by emission at energies $\mathrm{E}<10$ $\mathrm{keV}$. But a distribution of higher than average temperature regions can increase the average gas temperature observed at high energies. These regions with $T \gtrsim 10 \mathrm{keV}$ are observed also by XMM-Newton (Neumann et al. 2003) and Integral (Eckert et al. 2007). 
With reference to this interpretation, we notice that the value of $8.45 \mathrm{keV}$ in the HXD-PIN implies a temperature run that appears to be in disagreement with the XMM-Newton profile (Snowden et al. 2008; W09) as shown by the dot-dashed curve in Fig. 3. Moreover, as reported in the Introduction a temperature of $8.2 \mathrm{keV}$ has been derived combining data from Integral, $R X T E$ and ROSAT (Lutovinov et al. 2008).

An alternative and more likely explanation for the higher temperature value found in the joint $X M M-N e w t o n$ /HXD-PIN analysis may be given by the presence of the NT component itself in the spectrum which is responsible for the increase of the average temperature. A power law component in fact raises the exponential decline of the thermal emission, resulting in a higher best-fit thermal temperature. The poor fit with $T=8.2 \mathrm{keV}$ relative to the fit in which the temperature is a free parameter may be indicative of the presence of a second component in the Coma spectrum mainly visible in the energy range covered by the HXD-PIN data.

This SM analysis of the HXR spectrum in the Coma cluster confirms the results of the previous analysis by FF04. However, to remove the possibility that the existence of a NT excess in the HXR spectrum of the Coma cluster may be tied to the ICP average temperature value, we have considered the temperature profile (dot-dashed line in Fig. 3) that gives the average temperature of $8.45 \mathrm{keV}$ in the HXD-PIN FOV as reported by W09 in their joint XMM-Newton/Suzaku analysis. In this case, the PDS spectrum reports a c.l. for the excess of $\sim 4.3 \sigma$ and a NT flux of $1.15 \pm 0.41 \times 10^{-11} \mathrm{erg} \mathrm{cm}^{-2} \mathrm{~s}^{-1}$ in the energy range $20-80 \mathrm{keV}$ with $\Gamma=2$.

We examine also the possibility that the existence of the NT excess may depend on the temperature profile in the spatial range between $30^{\prime}$ and the virial radius $\left(R=78.24^{\prime}\right)$, up to now not covered by observations. To be conservative we have considered a flat temperature profile in this interval with a constant temperature of $\sim 7.8 \mathrm{keV}$ that is the value at $r=30^{\prime} \simeq 0.4 R$ (see Fig. 2). Although the flattening of the temperature profile seems to be very unlikely (see Fig. 3), in this case the excess is at the c.l. of $\sim 4.4 \sigma$. Finally, we have considered both these two effects: the higher average temperature of $8.45 \mathrm{keV}$ and the flat temperature profile at $r \geq 30^{\prime}$ with a constant temperature value of $\sim 8 \mathrm{keV}$. Also in these conditions the NT excess does not vanish in the HXR PDS spectrum though at the level of $\sim 3.8 \sigma$.

Independently from the real average temperature in the HXD-PIN FOV a relevant point emerges from the analysis of W09. They report that with a XMM-Newton average temperature of $8.2 \mathrm{keV}$, a nonthermal excess with c.l. greater than $4 \sigma$ is present in the Suzaku data, without including systematic effects, assuming a fixed photon index $\Gamma=2$ for the power law component. This result, absolutely in agreement with the PDS analysis (FF04), implies that the HXD-PIN spectrum is consistent with the PDS spectrum and therefore in disagreement with the PDS spectrum of RM04 that found a very marginal c.l. for the excess using the same temperature (8.21 $\mathrm{keV}$ ) and without considering systematic effects.

We also address the agreement between the Suzaku and BeppoSAX spectra with the following tests: a) we consider the smaller FOV of Suzaku HXD-PIN with respect to that of the PDS and 
temperature profile for an average $T=8.2 \mathrm{keV}$ (continuous and dashed lines in Fig. 1); in this case, we obtain a NT flux in the 20-80 keV energy band of $1.05 \pm 0.41 \times 10^{-11} \mathrm{erg} \mathrm{cm}^{-2} \mathrm{~s}^{-1}$ which is $\sim 20 \%$ lower than the PDS NT flux of $1.30 \pm 0.40 \times 10^{-11} \mathrm{erg} \mathrm{cm}^{-2} \mathrm{~s}^{-1}$ (see Sect. 2). The exclusion of part of the cooler cluster regions due to the smaller HXD-PIN FOV reduces the ICP contribution to the thermal emission mainly at energies around $15 \mathrm{keV}$. This determines a flatter ICP thermal spectrum and thus a lower NT excess that is at a c.l. of $\sim 4 \sigma$ in agreement with the W09 analysis.

b) we use the Suzaku FOV and temperature profile that gives an average $T$ of $8.45 \mathrm{keV}$ (dotdashed line in Fig. 3). In this case, the NT flux is $8.7 \pm 4.2 \times 10^{-12} \mathrm{erg} \mathrm{cm}^{-2} \mathrm{~s}^{-1}$ with a decrease of $\sim 33 \%$ with respect the PDS flux due to the smaller HXD-PIN FOV and the higher average temperature. This flux value is consistent with the upper limit reported by W09 of $6 \times$ $10^{-12} \mathrm{erg} \mathrm{cm}^{-2} \mathrm{~s}^{-1}$ for the NT spectral component with $\Gamma=2$.

This agreement between the Suzaku NT upper limit and the BeppoSAX detection has a further confirmation by Fig. 8 of Wik et al. (2011) when different spatial models are examined.

We believe that the NT flux of $1.05 \pm 0.41 \times 10^{-11} \mathrm{erg} \mathrm{cm}^{-2} \mathrm{~s}^{-1}$ (see case $a$ )) cannot be much different from the value measured by $\mathrm{W} 09$ for $\mathrm{T}=8.2 \mathrm{keV}$, and not reported in their paper (only the c.l. of the excess is in W09), because the flux determined for the case $b$ ) is consistent with the Suzaku upper limit.

Thus our SM analysis of the PDS spectrum reproduces the two results present in the W09 analysis: an excess at the c.l. of $\sim 4 \sigma$ for an average $X M M-N e w t o n$ temperature of $8.2 \mathrm{keV}$, and the upper limit for the NT flux with an average temperature of $8.45 \mathrm{keV}$ obtained by the joint XMM-Newton /Suzaku analysis. All this reinforces the consistency of the PDS and HXD-PIN spectra and therefore the presence of an hard tail in the Coma cluster spectrum.

\section{Conclusions}

For the first time the HXR spectrum of the Coma cluster has been analyzed using ICP temperature and density profiles instead to consider bremsstrahlung emission for an average temperature in the detector FOVs. These profiles are determined by the SM analysis of X-ray observables. This procedure has allowed to obtain further checks on the relevant point present in the Suzaku analysis that for a XMM-Newton temperature $T=8.2 \mathrm{keV}$ reports a NT excess at c.l. $\gtrsim 4 \sigma$ absolutely consistent with the results of FF04 (and therefore in disagreement with those of RM04). We have shown that the compatibility between the PDS and HXD-PIN spectra has a robust cross-check when in our SM analysis of the PDS data we consider the smaller HXD-PIN FOV and the different average temperatures of $8.2 \mathrm{keV}$ and $8.45 \mathrm{keV}$ (cases $a$ and $b$, respectively). The agreement between the two spectra is a further confirmation of the presence in the Coma cluster of a NT component. As reported in the previous Section, the PDS spectrum gives a hard excess with significance above $4 \sigma$ also for an ICP average temperature of $8.45 \mathrm{keV}$. This detection by the PDS is possible thanks 
to its FOV, a factor $\sim 4$ greater than the HXD-PIN FOV that instead reports a flux upper limit for the same temperature.

We thank Alfonso Cavaliere, Gianfranco Brunetti, Fabio Gastaldello, Francesco Lazzarotto and Fabio Pizzolato for helpful discussions and our referee for insightful comments, helpful toward improving our presentation. A.L. thanks SISSA for warm hospitality.

\section{REFERENCES}

Ajello, M., Greiner, J., Sato, G., et al. 2008, ApJ, 689, 666

Arnaud, M., et al. 2001, A\&A, 365, L67

Ajello, Rebusco, P., Cappelluti, N., Reimer, O., Bhringer, H., Greiner, J., Gehrels, N., Tueller, J., \& Moretti, A. 2009, ApJ, 690, 367

Bautz, M.W. et al. 2009, PASJ, 61, 1117

Bisnovatyi-Kogan, G.S. \& Pozanenko, A.S. 2010, astro-ph/1009.5564

Bonamente, M., Marshall, K.J., \& Lieu, R. 2003, ApJ, 585, 722

Bradač, et al. 2008, ApJ, 687, 959

Brown, S. \& Rudnick, L. 2010, astro-ph/1009.4258

Brunetti, G., Setti, G., Feretti, L., \& Giovannini, G. 2001, MNRAS, 320, 365

Castander, F.J., et al. 2001, AJ, 121, 2331

Cavaliere, A., \& Lapi, A. 2006, ApJ, 647, L5

Cavaliere, A., Lapi, A., \& Fusco-Femiano, R. 2009, ApJ, 698, 580

Cavaliere, A., Lapi, A., \& Fusco-Femiano, R. 2011, A\&A, 525, 110

Churazov, E., Sunyaev, R., Revnivtsev, M., et al. 2007, A\&A, 467, 529

Clowe, et al. 2006, ApJ, 648, L109

David, L.P., et al. 1993, ApJ, 412, 479

Eckert, D., Neronov, A., Courvoisier, T.J.-L., \& Produit, N. 2007, A\&A, 470, 835

Ferrari, C., Govoni, F., Schindler, S., Bykov, A.M., \& Rephaeli, Y. 2008, Space Science Review, 134,93

Frontera, F., Orlandini, M., Landi, R., Comastri, A., Fiore, F., Setti, G., Amati, L., Costa, E., Masetti, N., \& Palazzi, E. 2007, ApJ, 666, 86

Fusco-Femiano, R., dal Fiume, D., Feretti, L., Giovannini, G., Grandi, P., Matt, G., Molendi, S., \& Santangelo, A. 1999, ApJ, 513, L21 
Fusco-Femiano, R., Dal Fiume, D., De Grandi, S., Feretti, L., Giovannini, G., Grandi, P., Malizia, A., Matt, G., \& Molendi, S. 2000, ApJ 534, L7

Fusco-Femiano, R., Dal Fiume, D., Orlandini, M., Brunetti, G., Feretti, L., \& Giovannini, G. 2001, ApJ, 552, L97

Fusco-Femiano, R. Orlandini, M., Brunetti, G., Feretti, L., Giovannini, G., Grandi, P., \& Setti, G. 2004, ApJ, 602, L73 (FF04)

Fusco-Femiano, R., Landi, R., \& Orlandini, M. 2005 ApJ, 624, L69

Fusco-Femiano, R., Landi, R., \& Orlandini, M. 2007, ApJ, 654, L9 (FFLO07)

Fusco-Femiano, R., Cavaliere, A., \& Lapi, A. 2009, ApJ, 705, 1019 (FFCL09)

Gavazzi, R., et al. 2009, A\&A, 498, L33

George, M.R., Fabian, A.C., Sanders, J.S., Young, A.J., \& Russell, H.R. 2009, MNRAS, 395, 657

Gruber, D.E., Matteson, J.L., Peterson, L.E., \& Jung, G.V. 1999, ApJ, 520, 124

Hoshino, A., Henry, P.J., Sato, K., et al. 2010, PASJ, 62, 371

Hughes, J.P., Butcher, J.A., Stewart, G.C., \& Tanaka, Y. 1993, ApJ, 404, 611

Kaastra, J.S.,\& Mewe, R. 1993, A\&AS, 97, 443

Kawaharada, M., Okabe, N., Umetsu, K., et al. 2010, ApJ, 714, 423

Kirsch, M.G., et al. 2005, Proc. SPIE, 5898, 22

Kronberg, P.P., Kothes, R., Salter, C.J., \& Perillat, P. 2007, ApJ, 659, 267

Lapi, A., Cavaliere, A., \& Menci, N. 2005, ApJ, 619, 90

Lapi, A., Fusco-Femiano, R., \& Cavaliere, A. 2010, A\&A 516, L34

Leccardi, A., Rossetti, M., \& Molendi, S. 2010, A\&A, 510, A82

Lutovinov, A.A., Vikhlinin, A., Churazov, E.M., Revnivtsev, M.G., Sunyaev, R.A. 2008, ApJ, 687, 968

Mewe, R., Gronenschild, E.H.B.M., \& van den Oord, G.H.J. 1985, A\&AS, 62, 197

Mewe, R., Lemen, J.R. \& van den Oord, G.H.J. 1986, A\&AS, 65, 511

Mohr, J.J., Mathiesen, B., \& Evrard, A.E. 1999, ApJ, 517, 627

Morrison, R., McCammon, D., 1983, ApJ, 270, 119

Nakazawa, K., Sarazin, C.L., Kawaharada, M., Kawano, N., Fukazawa, Y., Inoue, S., Takizawa, M., Wik, D.R., Finoguenov, A., \& Clarke, T.E. 2009, PASJ, 61, 339

Neumann, D.M., Lumb, D.H., Pratt, G.W., \& Briel, U.G. 2003, A\&A, 400, 811

Rephaeli, Y. 1979, ApJ, 227, 364

Rephaeli, Y. \& Gruber, D. \& Blanco, P. 1999, ApJ, 511, L21

Rephaeli, Y. \& Gruber, D. 2002, ApJ, 579, 587 
Rephaeli, Y. \& Gruber, D. 2003, ApJ, 595, 137

Rephaeli, Y., Nevalainen, J., Ohashi, T., \& Bykov, A.M. 2008, Space Science Reviews, Vol. 134, Issue 1-4, pp. 71-92

Reiprich, T.H., Hudson, D.S., Zhang, Y.-Y., et al. , A\&, 501, 899

Ricker, P.M., \& Sarazin, C.L. 2001, ApJ, 561, 621

Rossetti, M. \& Molendi, S. 2004, A\&A, 414, L41 (RM04)

Snowden, S.L., Mushotzky, R.F., Kuntz, K.D., \& Davis, D.S. 2008, A\&A, 478, 615

Vikhlinin, A, Kravtsov, A., Forman, W., Jones, C., Markevitch, M., Murray, S.S., \& Van Speybroeck, L. 2006, ApJ, 640,691

Wik, D.R., Sarazin, C.L., Finoguenov, A., Matsushita, K., Nakazawa, K., \& Clarke, T.E. 2009, ApJ, 696, 1700 (W09)

Wik, D.R., Sarazin, C.L., Finoguenov, A., Baumgartner, W.H., Mushotzky, R.F., Okajima, T., Tueller, J., \& Clarke, T.E. 2011, ApJ, 727, 119 

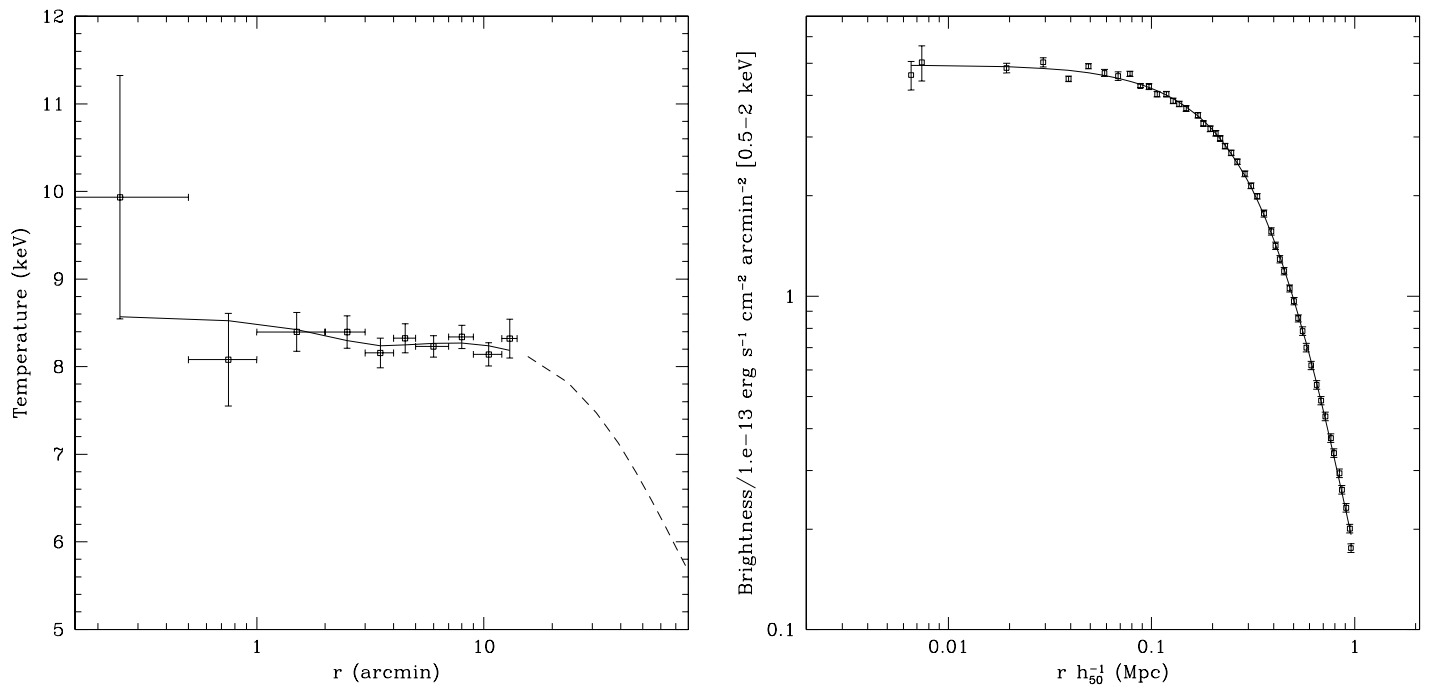

Fig. 1. - left panel: Projected emission-weighted temperature profile measured by XMMNewton (Snowden et al. 2008). The continuous line represents the SM fit (see FFCL09). The dashed line is the extrapolation of the SM fit to the virial radius $R$. This profile gives an average temperature of $8.21 \pm 0.08 \mathrm{keV}\left(90 \%\right.$ c.l.) in the HXD-PIN FOV $\left(34^{\prime} \times 34^{\prime}\right)$, the same value reported by W09 in their XMM-Newton analysis of the Coma cluster; right panel: Brightness profile in the energy range 0.5-2 keV measured by ROSAT (Mohr et al. 1999). The continuous line represents the SM fit $\left(\chi^{2}=55.5 / 44\right)$. 

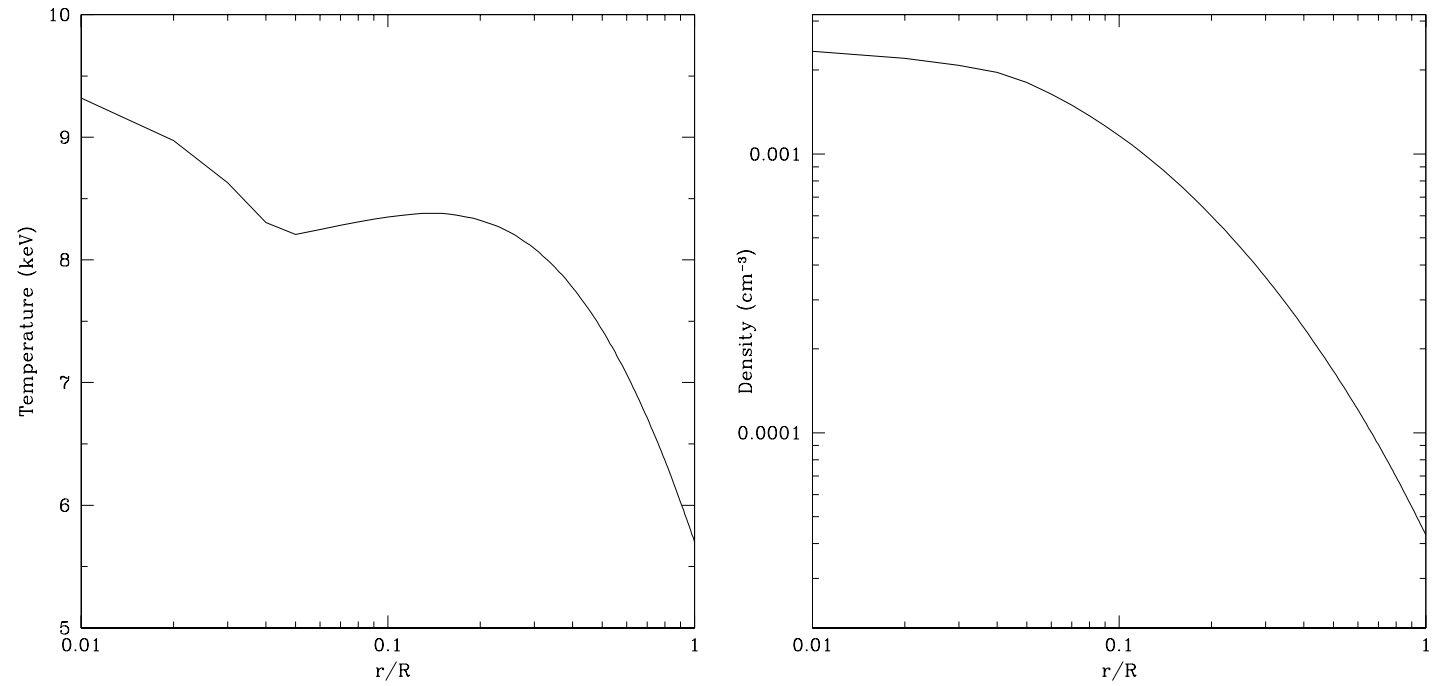

Fig. 2.- left panel: Temperature profile that fits the projected emission-weighted temperature profile of Fig.1; right panel: Density profile that fits the brightness profile of Fig. 1. 


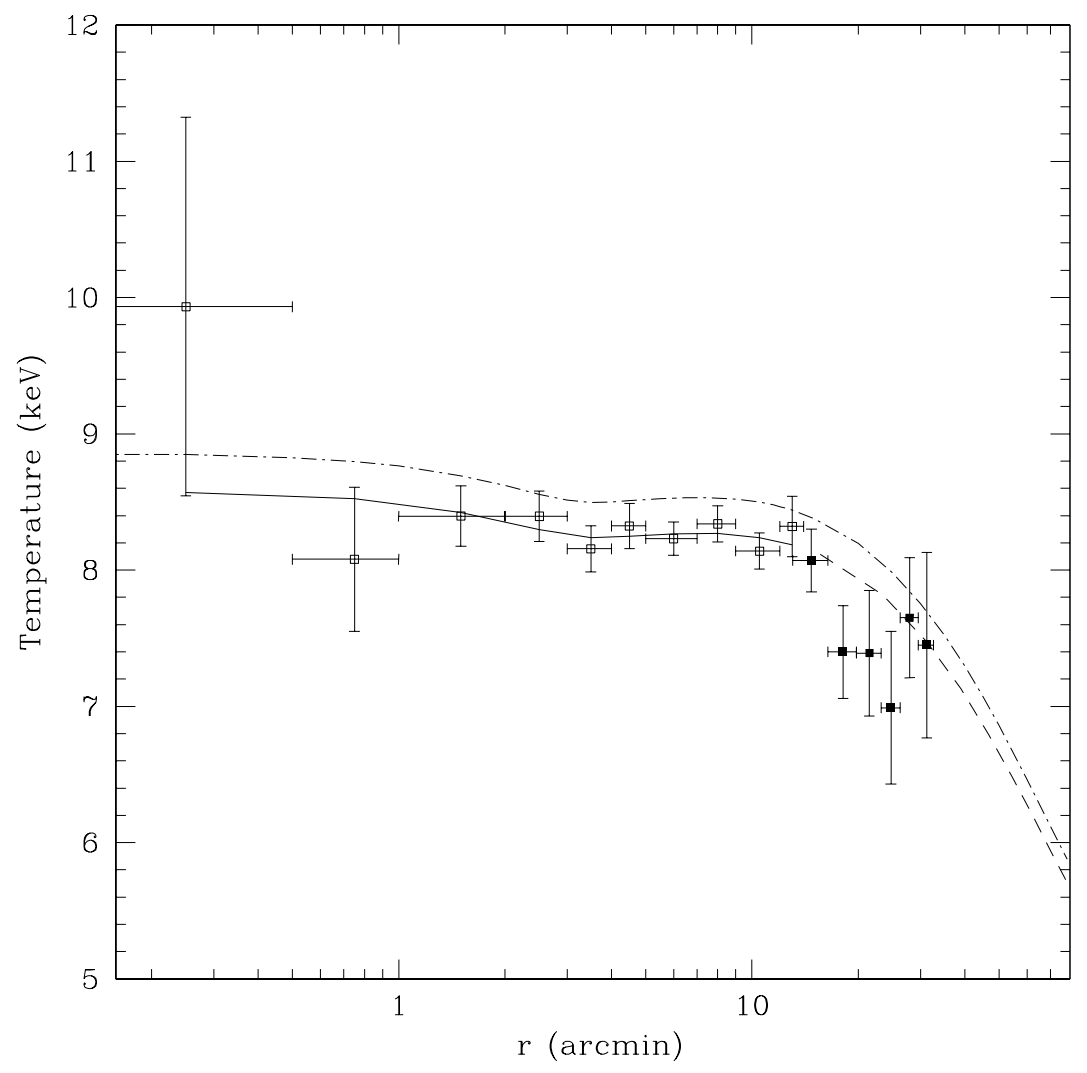

Fig. 3. - The continuous line represents the SM fit to the XMM-Newton temperature profile (empty square points, Snowden et al. 2008) and the dashed line is the SM extrapolation to the virial radius (see Fig. 1); the dot-dashed line is the SM fit with an average temperature of $8.45 \mathrm{keV}$ in the HXD-PIN FOV reported by the joint XMM-Newton / Suzaku analysis (W09). The filled square points are the temperature values in the XMM-Newton regions R1-R6 investigated by W09. 


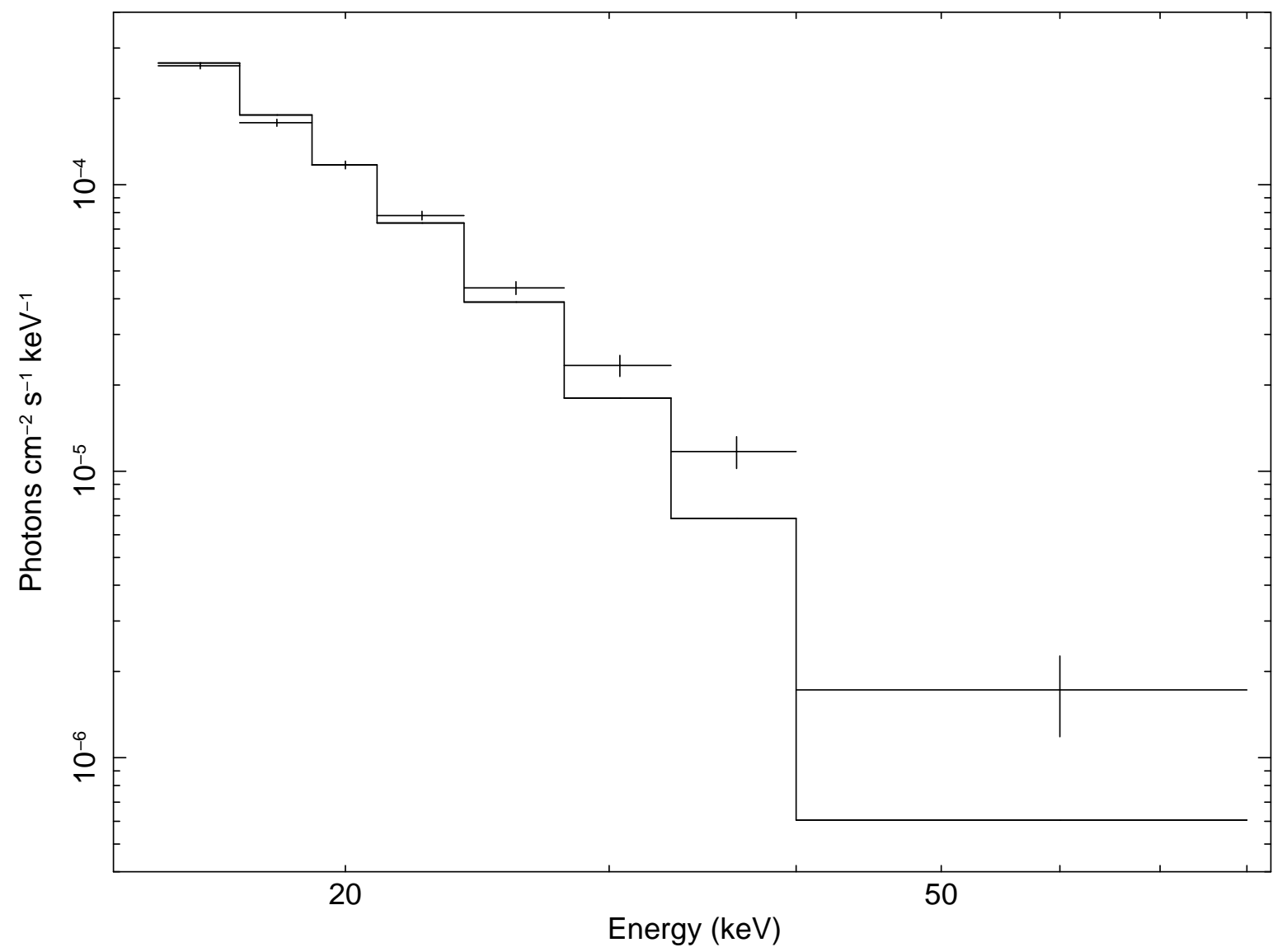

Fig. 4. - The data represent the HXR spectrum of the Coma cluster observed by BeppoSAX /PDS (FF04). The continuous line is the thermal ICP emission derived from the SM analysis using the temperature and density profiles of Fig. 2. 


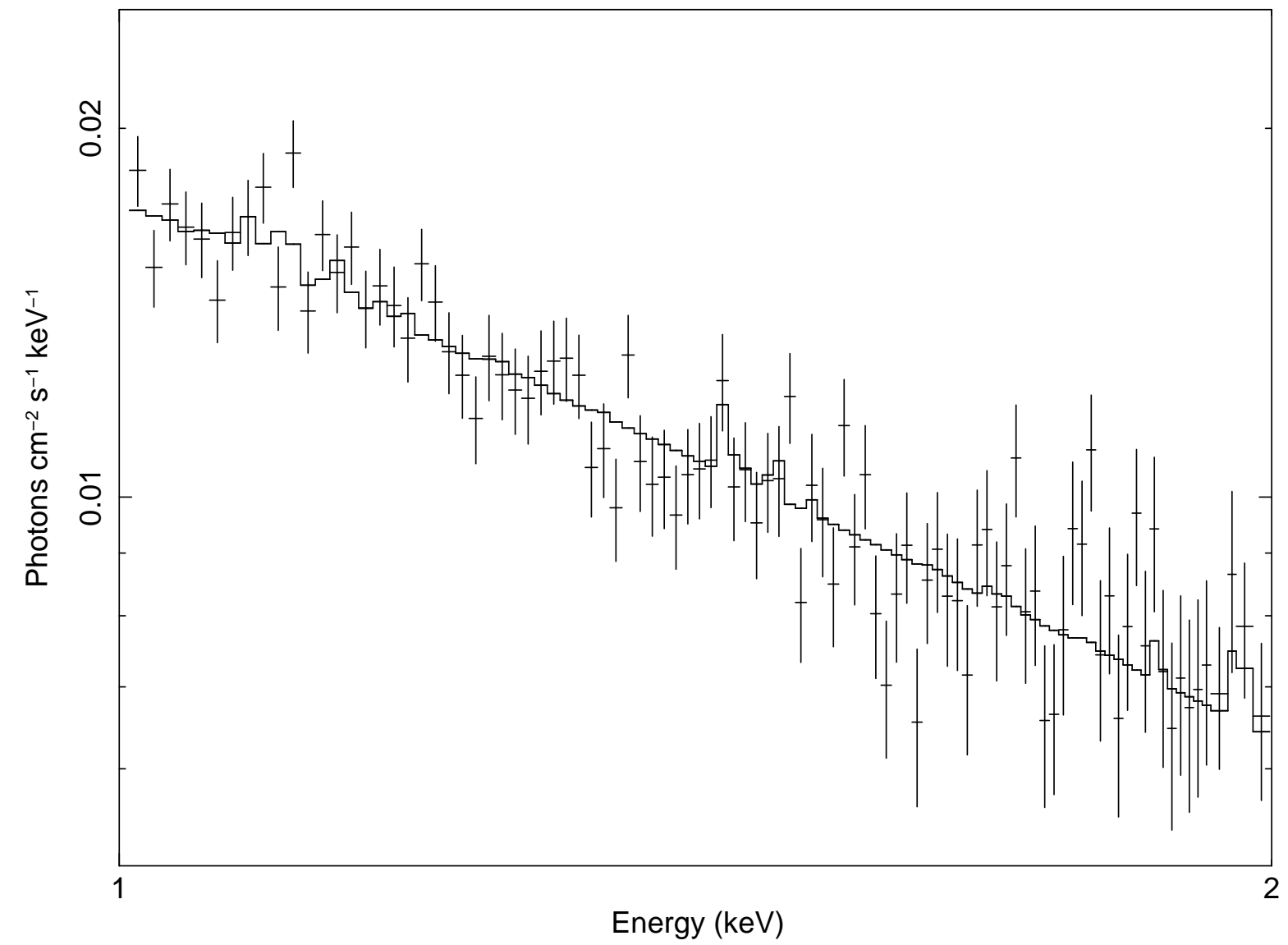

Fig. 5.- ROSAT PSPC spectrum of the $20^{\prime}-40^{\prime}$ region around the center of the Coma cluster, fitted with the SM profiles of Fig.2 $\left(\chi^{2}=105.85 / 99\right)$. 